\title{
BMJ Open 'Has she seen me?': a multiple methods study of the pharmaceutical care needs of older people with sensory impairment in Scotland
}

\author{
Nour Alhusein, ${ }^{1}$ Leah Macaden, ${ }^{2}$ Annetta Smith, ${ }^{2}$ Kathleen M Stoddart, ${ }^{3}$ \\ Andrea J Taylor, ${ }^{1}$ Kirsty Killick, ${ }^{4}$ Thilo Kroll, ${ }^{5}$ Margaret C Watson ${ }^{1}$
}

To cite: Alhusein N, Macaden L, Smith A, et al. Has she seen me?': a multiple methods study of the pharmaceutical care needs of older people with sensory impairment in Scotland. BMJ Open 2018;8:e023198. doi:10.1136/ bmjopen-2018-023198

- Prepublication history and additional material for this paper are available online. To view these files, please visit the journal online (http://dx.doi org/10.1136/bmjopen-2018023198).

Received 26 March 2018 Revised 13 July 2018 Accepted 13 July 2018

Check for updates

(C) Author(s) (or their employer(s)) 2018. Re-use permitted under CC BY-NC. No commercial re-use. See rights and permissions. Published by BMJ.

${ }^{1}$ Department of Pharmacy and Pharmacology, University of Bath, Bath, UK

${ }^{2}$ Department of Nursing, Centre for Health Science, University of the Highland and Islands, Inverness, UK

${ }^{3}$ Faculty of Health Sciences and Sport, University of Stirling,

Stirling, UK

${ }^{4}$ School of Health and Social Care, Edinburgh Napier University, Sighthill Campus, Edinburgh, UK

${ }^{5}$ School of Nursing, Midwifery and Health Systems, University College Dublin, Dublin, Ireland

Correspondence to Dr Margaret C Watson; M.C.Watson@bath.ac.uk

\section{ABSTRACT}

Objectives To explore the pharmaceutical care needs of, and service provision to, older people with sensory impairment (visual, hearing and dual impairment) on prescribed polypharmacy ( $\geq 4$ medicines) in Scotland. Design Interviews were conducted with older people with sensory impairment and community pharmacy personnel, which informed the content of a subsequent national cross-sectional survey of community pharmacists.

Setting Scotland, 2015-2016.

Participants Older people with sensory impairment and community pharmacy personnel.

Results Interviews were completed with 23 older people with sensory impairment (dual impairment $n=13$, visual or hearing impairment $n=5$ of each) and 30 community pharmacy personnel from eight of 14 Scottish Health Boards. A total of 171 survey responses were received. Older people reported that they did not always disclose their sensory impairment to pharmacy personnel. They also reported that medicines were difficult to identify particularly when their name, shape or colour changed. Pharmacy personnel relied on visible cues such as white canes or guide dogs to identify visual impairment and suggested that hearing loss was less visible and more difficult to identify. Many assistive aids in support of medicine management, such as dosette boxes, seemed inadequate for complex medication regimens. Few community pharmacy personnel reported receiving training in the care of people with sensory impairment.

Conclusions This is the first comprehensive, multistakeholder, in-depth exploration of the pharmaceutical care needs of older people with sensory impairment. Strategies are needed to enable people with sensory impairment to disclose their impairment to pharmacy personnel (and other healthcare providers). Community pharmacy personnel require training to deliver person-centred pharmaceutical care for older people with sensory impairment particularly regarding communication with individuals in this vulnerable population.

\section{INTRODUCTION}

Sensory impairment affects any age group but is one of the most common long-term conditions of later life. ${ }^{1}$ Hearing and vision loss are
Strengths and limitations of this study

- This study adopted a multiple methods approach with the qualitative elements providing rich data on the topics addressed.

- The low response rate to the national survey, limited the generalisability of the results.

- The nature and scope of identified barriers and facilitators to the receipt or provision of pharmaceutical care were exemplified in the interviews both with patients and community pharmacy personnel, respectively.

on the increase globally and are becoming an important consideration as populations age. ${ }^{2}$ Recurrent experiences of communication breakdown occur among people with sensory impairment and are associated with negative psychosocial well-being and reduced social participation activities. ${ }^{3-5}$ Older people with hearing, visual and dual impairment have higher rates of comorbidities and activity limitations than older people without impairment. ${ }^{6}$ Additionally, the majority of individuals $>75$ years use at least three prescribed medicines on a regular basis. ${ }^{7}$ This use of multiple medicines, also known as polypharmacy, is increasing as a result of the multimorbidity associated with an ageing population. ${ }^{8}$ Thus, appropriate pharmaceutical care, that is, 'the pharmacist's contribution to the individuals' care in order to optimise medicine use and improve health outcomes', ${ }^{9}$ particularly for those with polypharmacy needs is central to the management of multimorbidity. ${ }^{8}$

The United Nations Convention on the Rights of Persons with Disabilities,${ }^{10}$ stipulates that nations 'Provide persons with disabilities with the same range, quality and standard of free or affordable healthcare and programmes as provided to other persons...' This means that people with sensory impairment are entitled to the same 
range, quality and standard of pharmaceutical care as well and that necessary accommodations have to be made.

However, little is known about the pharmaceutical care experiences of older people with sensory impairment. There is a need to be aware of patients' needs in order to deliver pharmaceutical care to this vulnerable patient population. Difficulties have been reported in managing medications, ${ }^{6}$ reading labels, ${ }^{11} 12$ identifying expiration dates $^{1213}$ and distinguishing between different tablets. ${ }^{14}$ Individuals with visual impairment often rely on memory to distinguish between medicines or on daily help to administer/organise their medicines, ${ }^{14}$ while individuals with hearing impairment can struggle with the complexity of written medicines information. ${ }^{14}$

Few studies have explored the provision of pharmaceutical care by pharmacy personnel to patients with sensory impairments. ${ }^{15} 16$ A recent scoping review (conducted by the authors of this study) identified 11 studies which demonstrated that people with sensory loss had lower levels of medication knowledge and were at higher risk of iatrogenic harm compared with people without sensory loss. ${ }^{16}$ For example, one cross-sectional survey evaluating practising pharmacists' attitudes towards and methods of interacting with deaf patients in the USA reported that two-thirds of community pharmacy personnel were not comfortable interacting with patients with hearing impairment because of communication difficulties. ${ }^{17}$ Commonly used methods for communication with individuals with hearing or visual impairment included hearing loops and large print resources, respectively. ${ }^{18}$

The aim of this current study was to undertake a comprehensive exploration of the:

- Pharmaceutical care needs of older people with sensory impairment who were receiving polypharmacy.

- Experiences, attitudes and training needs of community pharmacy personnel regarding the provision of pharmaceutical care to this patient population.

\section{METHODS}

A multimethod approach was used comprising: semistructured telephone or face-to-face interviews with older people (depending on their preference) with sensory impairment on polypharmacy (hereafter referred to as patients); semistructured telephone interviews with community pharmacy personnel (ie, pharmacists, pharmacy assistants) and a national cross-sectional survey of community pharmacists. The purpose of the interviews and survey was to characterise experiences, barriers and facilitators to the provision pharmaceutical care for older people with sensory impairments for patients with sensory impairment from different perspectives.

\section{Sampling and recruitment}

\section{Interviews}

Older people with sensory impairment were recruited through different organisations including Forth Valley Sensory Centre, Perth Macular Society, Action on Hearing Loss and North East Sensory Services. The emails were sent on behalf of the interviewer (KK) to potential participants by the above centres. Interested participants then contacted the researcher. Additionally, the study was advertised by Sight Action (a charity which offers support to blind partially sighted people in the Scottish Highlands and Islands) in their newsletter.

Community pharmacy personnel were recruited using a variety of methods. The study was advertised through Community Pharmacy Scotland (CPS), ${ }^{19}$ the Scottish national community pharmacy contractor organisation. Participants were also recruited at a training event which included a session on caring for patients with hearing impairment. Stratified sampling was undertaken of respondents to reflect geographical variation (urban and rural) and pharmacy type (independent, small chain, large multiple). Snowball recruitment ${ }^{20}$ was also used, that is, participants were asked to recommend other colleagues to participate. Informed consent was obtained from all participants. All interviews were conducted by a postdoctoral female health psychologist researcher (KK).

Topic guides (online Supplementary appendices 1 and 2) were developed by the project team and the Project Advisory Group (PAG). The PAG consisted of representatives of visual and hearing impairment organisations, such as Action on Hearing Loss, Sight Action and Age Scotland and provided advice and guidance to the team throughout the project on research methodology and procedures (including the ethics application, interview schedules and survey development). Topic guides were pilot tested and no changes were required. The data generated from pilot interviews were included in the final analysis. The interviews took place between 2015 and 2016 in Scotland. Patient interviews included exploration of: their methods of managing their medicines, the support they received in relation to their medicines, their use of community pharmacies, barriers and facilitators to their use of medicines and community pharmacies and whether pharmacy personnel were aware of the patient's sensory impairment. The pharmacy personnel interviews explored: current provision of pharmaceutical care to older people with sensory impairment, including barriers and facilitators to care provision, perceptions about medication adherence of this patient population and their training needs in providing pharmaceutical care to these patients.

\section{Cross-sectional survey}

The purpose of the survey was to explore community pharmacists' experience of, and attitudes towards, the provision of pharmaceutical care to older patients with sensory impairment, as well as to identify any training needs in relation to the provision of care to this patient population. The content of the questionnaire was informed by the emergent themes of preliminary analysis of interviews with pharmacy personnel and consultation with the PAG. The questionnaire comprised 24 questions using Likert-type scales, multiple choice questions and one open-ended question. The questions captured 
respondent demographics and explored their past and current experiences with providing pharmaceutical care to people with visual, hearing and dual impairments; training to cater for this patient group; perceived barriers and facilitators to providing pharmaceutical care and pharmacy facilities aimed at the needs of patients with sensory impairment, for example, hearing loop, Braille labels. In November 2016, the paper-based copy of the questionnaire was mailed to every community pharmacy $(\mathrm{n}=1186)$ in Scotland.

\section{Data management and analysis}

All interviews were digitally recorded, transcribed and anonymised. The first five interviews (of each cohort that is, patients, then community pharmacy personnel) were subjected to duplicate, independent coding to assess consistency and a coding framework was developed inductively from the data (NA and AT) ${ }^{21}$ The framework descriptions were finalised following discussion with the research team. All remaining transcripts were then coded by one researcher (NA) and analysed using thematic anal$\mathrm{ysis}^{22} 23$ in NVivo (V.11). Data saturation was achieved with no new themes emerging in the later interviews. Survey data were manually entered into SPSS V.22 and independent, duplicate checking was performed. Descriptive analyses were undertaken.

\section{Public and patient involvement}

An advisory group was established which consisted of representatives with sight and/or hearing loss and relevant patient organisations, such as Action on Hearing Loss, Sight Action and Age Scotland, which provided advice and guidance to the project team on research methodology and procedures and recruitment. Their support was particularly useful in the development and implementation of appropriate, accessible materials and practices throughout the project (including the ethics application, interview schedules and survey development).

\section{Ethics}

NHS ethical approval was not required because patients were not recruited through NHS organisations.

\section{RESULTS}

\section{Sample demographics}

Twenty-three older people with sensory impairment took part in the interviews. They had been recruited from six Health Board areas. Fifteen interviewees were female, the average age was $81 \pm 8$ years (range $67-95$ years) and 14 lived alone. The majority $(n=13)$ reported dual impairments and five individuals each had visual or hearing impairments, respectively. Interviews lasted an average of $28 \pm 11 \mathrm{~min}$.

Thirty community pharmacy personnel, 17 of whom were pharmacists, took part in semistructured interviews. Just over half of $(n=17)$ interviewees worked in independent, single outlet pharmacies. Interviewees were recruited from eight of the 14 Scottish Health Boards and interviews lasted an average of $14 \pm 6 \mathrm{~min}$.

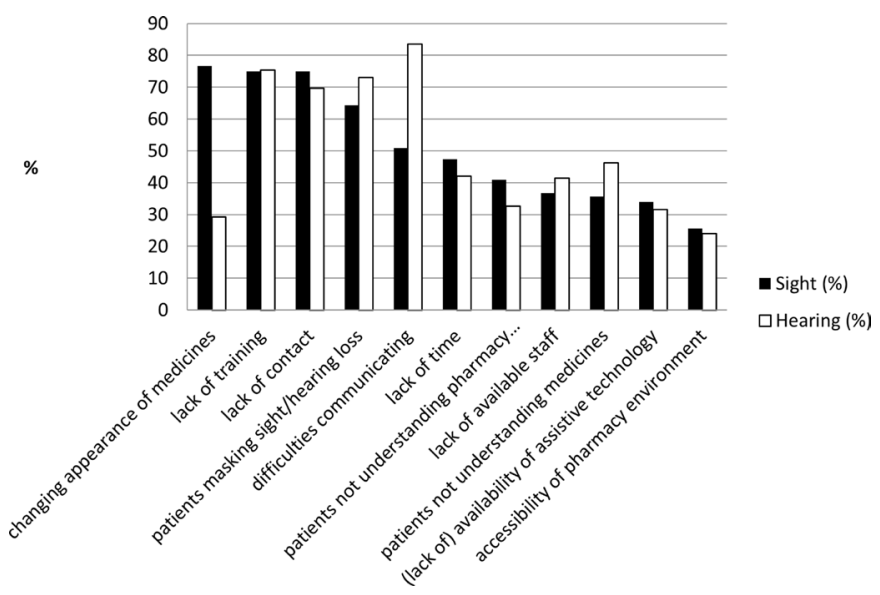

Figure 1 Survey respondents' perceived barriers to providing care for patients with visual or hearing impairment.

Completed questionnaires were returned from $171 / 1186$ (14.4\%) pharmacy personnel. Most respondents $(>85 \%) \quad(\mathrm{n}=143)$ reported providing pharmaceutical care for people with visual or hearing impairment and $39 \%(\mathrm{n}=66)$ reported providing care for people with dual impairments.

\section{Factors affecting accessing to, and provision of, pharmaceutical care}

A wide range of barriers (figure 1) and facilitators (figure 2) were identified by both types of interviewees and survey respondents in terms of accessing and providing pharmaceutical care to patients with sensory impairment. These factors influenced all stages of the pharmaceutical care journey (figure 3), that is, from the ordering or supply of medicines to their storage and administration, the most dominant of which are presented below. Any failure or weakness in the pharmaceutical care journey was associated with communication difficulties and safety concerns, which in turn lead to suboptimal pharmaceutical care.

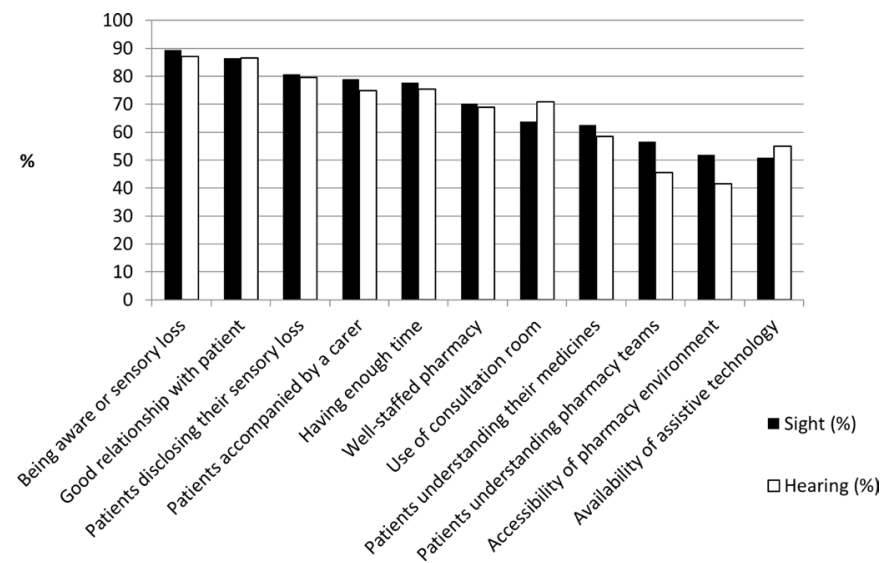

Figure 2 Survey respondents' perceived facilitators to providing care for patients with visual or hearing impairment. 


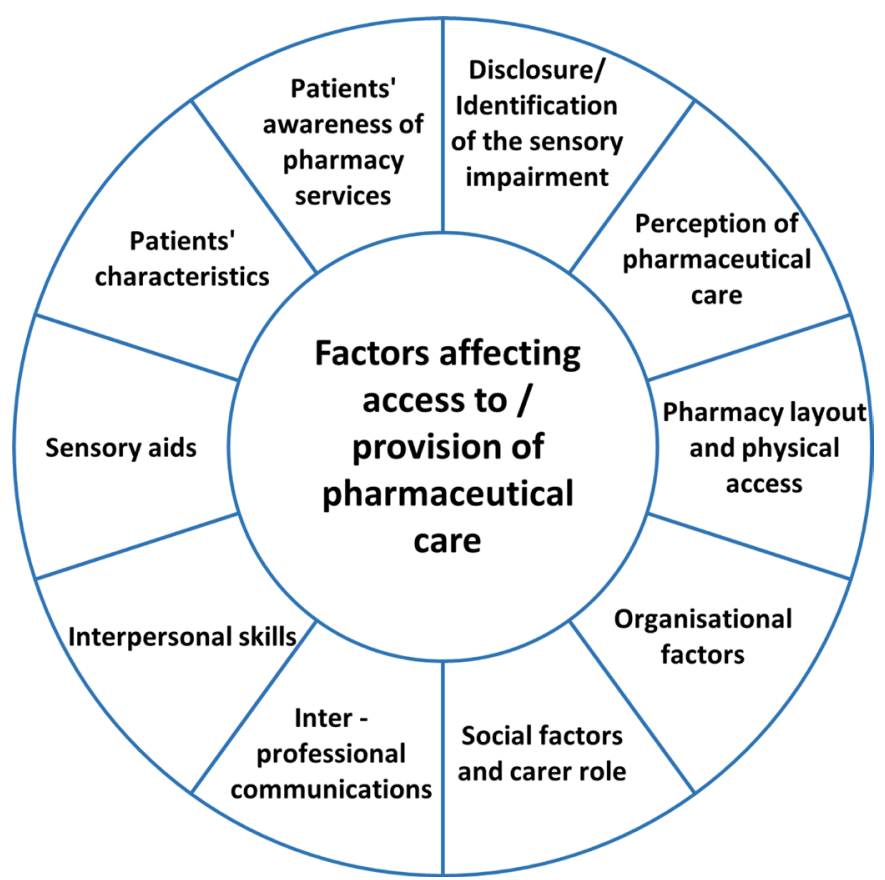

Figure 3 Factors affecting access to/provision of pharmaceutical care to older people with sensory impairment.

\section{Ordering and accessing medicines}

Older people with sensory impairments reported challenges with obtaining prescribed medicines which varied according to the method of ordering, that is, in person in the pharmacy, by phone, online or home delivery services. Difficulties included, patients having to make repeated visits to obtain their medications and waiting lists to receive a delivery service. Patients gave examples of the challenges that they encountered.

You would stand there [in the pharmacy]-has she seen me? Or you've to come back in twenty minutes and you're standing there. You don't know-have they got it ready, have they seen me? (ID2, Female, Dual impairment)

They can sometimes get confused or they won't hear if you've shouted out their named prescription (ID26, Pharmacist, Multiple chain)

\section{Pharmacy environment}

Some participants with visual loss also reported that the layout of pharmacies was difficult to navigate.

You've got shelves on either side with things displayed on it and you're likely to bump into that and send things flying onto the floor... (ID1, Male, Dual impairment)

Ordering online or by phone solved the problem of repeated visits for older people with sensory impairment, that is, they did not need to physically attend a pharmacy to order/receive their medication. One patient described how access to a large visual screen helped him order his prescription.
There must be many people again who can't use a computer. I mean I've got a 32-inch screen I use for my computer (ID5, Male, Dual impairment)

However, while home delivery services helped patients avoid the need to travel to the pharmacy, pharmacy personnel were concerned that it reduced the opportunity for direct communication with patients.

The other problem is even with some of the independent pharmacies, these patients are now being cared for with delivery services... So it's more the delivery driver who sees them... rather than the pharmacist (ID1, Pharmacist, Supermarket)

\section{Communication about ordering and accessing medicines}

While patients also valued the opportunity for direct contact with pharmacy personnel and the opportunity that this afforded to discuss their medicines, they also cited difficulties in communicating with personnel.

I'd like to see some sort of annual review at least... you've been invited for an interview with the pharmacist. They could go through your drug requirements with you (ID13, Male, Hearing loss)

People with hearing impairment reported that they did not always hear the full administration instructions, and this could be exacerbated by enunciation/accent in telephone conversations.

It was the tail end of the sentence before I had worked out what she was talking about and I didn't know which tablet she was talking about... you have a slight moment of panic of, am I going to have to ask her to repeat (ID15, Female, Hearing loss)

Pharmacy interviewees also discussed the challenges for communicating administration information to hearing impaired patients.

Three of them [patients] have quite bad hearing loss and they are all on polypharmacy, so it is quite difficult to communicate with any of them regarding any issues that comes up with their medication (ID3, Preregistration Pharmacist, Small chain)

I suppose with the 'seeing ones' [sic: meaning patients with visual impairment] it's more you can't really give them the patient information leaflet (ID20, Pharmacist, Independent)

Patients identified interventions which helped to improve communication including: one-to-one conversations, where the individual spoke slowly and clearly (particularly with lip reading); use of sign language and the provision of written instructions (but this was not considered very helpful by those with visual impairment).

For patients with dual impairment, one participant mentioned having an interpreter and using the deafblind manual. 
The other thing of course you've got deaf-blind manual which is doing the alphabet on your hand which is slow but just as effective anyway (ID1, Male, Dual impairment)

Survey respondents perceived that difficulties in communication were a much greater barrier when providing care to patients with hearing impairment $(>80 \%, \mathrm{n}=143)$ compared with visual impairment $(50 \%, \mathrm{n}=87)$. While pharmacy personnel interviewees discussed hearing and visual impairment as separate entities, there was little acknowledgement of dual impairment. Where dual impairment was identified, there was strong reliance on the carer to manage patients' medications (if they had a carer).

\section{Managing and storing medicines}

\section{Inability to distinguish medicines}

Patients with visual impairment reported difficulties when differentiating between tablets, reading instruction leaflets and expiry dates. The majority $(70 \%, \mathrm{n}=131)$ of survey respondents also cited that the changing appearance of medication or packaging as a problem for patients with visual impairment.

It's a bit of guess work really...I don't read anything on them but there's one packet that's got red stripes. The difficulty there of course is when the pharmacy orders from a different drug company and it's a change of packet (ID7, Female, Dual impairment)

\section{Assistive medicine management devices}

Provision of large written font on labels and Braille instructions on boxes helped; however, medicine labels were sometimes placed over Braille and large print labels had insufficient space for detailed instructions.

Patients reported difficulties in using monitored dosage systems and other packaging, such as pushing tablets out of foil wrapping and some described developing their own strategies to manage these types of storage systems.

If it's simply a case of these little things with seven little boxes I can get somebody to do my own pills and put them in one of these (ID3, Female, Visual loss)

It was a blister pack where she pops them out and half of them fall onto the floor: there are no more tablets in the house. They are lost (ID21, Pharmacist, Independent)

\section{Safety concerns}

\section{Disclosure and identification of sensory impairment}

A major barrier to the access and provision of care, which was identified, was related to disclosure by patients and/ or awareness by pharmacy personnel, of the presence of a sensory impairment, which may compromise safety. Many patients were unsure that their pharmacist was aware of their impairment and if they were aware, this was attributed to the presence of visual/physical cues such as a guide dog or hearing aids. Few $(12 \%, n=21)$ survey respondents reported having a process to identify people with sensory impairment.

I've just always managed. Sometimes I wonder if my doctor's practice realises I'm going blind. Sometimes I don't think they're aware of it (ID6, Male, Dual impairment)

It wouldn't necessarily be obvious that somebody has an impairment of some description (ID20, pharmacist, Independent)

Survey respondents $(70 \%, \mathrm{n}=117)$ perceived that individuals with visual impairment asked for help with their medicines more often than individuals with hearing impairment $(33 \%, \mathrm{n}=55)$. The respondents had greater concerns $(>90 \%, \mathrm{n}=158)$ about people with visual impairment managing their medicines than hearing impairment $(>75 \%, \mathrm{n}=126)$.

Community pharmacy personnel described many safety concerns such as the wrong medicines being taken at the wrong time, inappropriate dose and patients' lack of understanding of medicine indication and possible side effects. Study participants reported safety incidents which occurred due to communication difficulties.

They had reintroduced the $4 \mathrm{mg}$ tablets and I was taking $8(4 \mathrm{mg})$ tablets a day instead of 8 (2mg) tablets so my blood pressure just went right down (ID5, Male, Dual impairment)

We had a patient, I think he is on at least 15 or 16 different medications. He was getting quite bad side-effects from his pain relief medication, so dizziness and I think he reported falling over once ... it was quite an urgent matter and it is quite difficult getting all of his personal information from him (ID3, Preregistration Pharmacist, Small chain)

We couldn't explain to her the need to use these ones carefully and not become addicted to them... I think maybe if she had been hearing or it was [sic, it would have been] easier for us to communicate (ID1, Pharmacist, Supermarket)

Improving the quality of pharmaceutical care for patients with sensory impairment

Education and training

Community pharmacy interviewees and survey respondents reported that they had insufficient training to support the needs older people with sensory impairment. Few (14\%, $\mathrm{n}=24$ ) survey respondents reported having received training to support older patients with visual or hearing impairment (8\%; $n=14$ for dual impairment). They reported that pharmacy support personnel had received less training than the pharmacists. The majority $(>80 \%, \mathrm{n}=138)$ of respondents stated that they would like additional training. Pharmacy personnel suggested that the national Chronic Medication Service (during which patients' medication is reviewed), ${ }^{24}$ provided an opportunity to speak to patients, including older patients with sensory impairment, and to undertake medication reviews. 
One of the things we do check is... how is their visual [sic] and do they have any problems reading labels or getting information from leaflets (ID24, Pharmacist)

\section{Assistive medicine management support}

Some participants described specific approaches to providing pharmaceutical care for this patient population, for example, the preparation of special medicine boxes and counselling at home to simplify complex dosing schemes. They acknowledged, however, the need to personalise the service provided.

But I guess the starting point with somebody with a sensory issue would be finding out how they would prefer the information (ID15, Pharmacist, Independent)

\section{DISCUSSION}

This study is the first exploration of pharmaceutical care provision to older people with sensory impairments in the UK. Data were obtained from 53 interviews: older people with sensory impairment (23) and community pharmacy personnel (30) across Scotland. One in seven pharmacies in Scotland were represented in the survey. The key themes that emerged included barriers and facilitators in relation to ordering and accessing medicines and in terms of managing medicines appropriately. Non-identification of sensory impairment or non-disclosure as well as various communication and accessibility challenges were perceived as possible safety hazards.

\section{Barriers and facilitators to accessing and providing pharmaceutical services}

Barriers and facilitators to access and provision of pharmaceutical services by and for older people with sensory impairment are illustrated in figures 2 and 3. These results could be used to improve pharmaceutical care of this patient population. A particular challenge identified was that a sensory impairment was not necessarily evident to community pharmacy personnel and this may be further complicated in that people with sensory impairment may not disclose vision or hearing loss. Furthermore, some patients indicated a preference for ordering their medicines online or by telephone, and this could limit the opportunities for community pharmacy personnel to interact/communicate with patients about their pharmaceutical care. Additionally, of the pharmacies represented in this study, few had specific processes to identify people with sensory impairment. This observation is also supported by findings of a recent study of pharmacists from different sectors in England. ${ }^{11}$ The lack of visibility of sensory impairment(s) means that the pharmaceutical care cannot be tailored to meet the individual patient's requirements. It has also been reported that most patients with visual impairment rely on others to obtain their medicines. ${ }^{25}$

\section{Safety and communication}

Similar to other countries, the current quality strategy (Prescription for Excellence) $^{26}$ in Scotland, emphasises the provision of safe, effective and person-centred pharmaceutical care to people. Our study identified several areas where community pharmacy personnel had concerns about medicine safety for older people with sensory impairment. Patients indicated the importance of informing them if a change in their medication regimens occurred. It would therefore be appropriate for community pharmacy personnel to both recognise this need and support patients as required to identify their medicines. ${ }^{11}$

Evidence suggests that patients with hearing impairment/s often do not understand the information given to them about their medications ${ }^{27}$ and sometimes may give the impression that they have understood medicine advice given to them. ${ }^{14}$ Pharmacists have previously indicated that their preferred choice of communication with this patient group is in writing ${ }^{17}$ but this might not reflect individual patient's preferences. Ideally, community pharmacy personnel should endeavour to provide information about each patient's medicines in a format that is acceptable and accessible to the individual patient. Older people with sensory impairment might also suffer from other cognitive impairments, for example, dementia. ${ }^{28}$ While we did not assess or explore cognitive function in this study, both pharmacy personnel and patients reported difficulties in retaining medicines' information for people with memory loss or dementia. These difficulties were compounded for people with sight loss, as they are less likely to be able to use written material as memory prompts.

\section{Improving the quality of pharmaceutical care for patients with} sensory impairment

There is a need to develop bespoke strategies to engender the recognition of sensory impairment by community pharmacy personnel and disclosure by older people with sensory impairment. A previous multicentre study which aimed to measure the outcomes of a structured programme provided to elderly patients by community pharmacists found that the intervention programme had some positive effects on satisfaction with treatment, sign and symptom control and economic outcomes. ${ }^{29}$ The multicentre international study was performed in seven European countries. The intervention comprised patient assessment, formulating an intervention and monitoring plan for each individual patient. Pharmacy interventions included: (1) educating the patient about their drug regimen and their medical condition, (2) implementing compliance-improving strategies such as drug reminder charts and (3) rationalising and simplifying drug regimens in collaboration with the patient's general practitioner.

Older people also need to be empowered to ask about medication regimens and contribute to the development of systems that facilitate and enable their communication between community pharmacy personnel (and other healthcare professionals). ${ }^{29}$ Involving patients in service improvement and health professional education 
and training plays a key part in the redesign of healthcare delivery. ${ }^{30}$ Our study demonstrates the unique experience of the older person with sensory impairment and the need to inform future strategies to remove barriers and enhance accessibility, equity and quality of services.

Community pharmacy personnel need to provide information in suitable, alternative formats, minimise (where possible) and explain changes in medication appearance to service users, and perform regular medicine reviews using a patient-centred approach to provide safe and effective pharmaceutical care. Future research needs to be targeted at creating innovative medication dispensing aids that reflect polypharmacy needs with irregular changes and adjustments in dosage. The pharmacy environment needs to be modified and adapted to accommodate service users with sensory impairment. Ideally, modifications should be guided by design experts, evidence based and in collaboration with older people with sensory impairment.

Community pharmacy personnel identified a need for evidence-informed education and training in the provision of pharmaceutical care to older people with sensory impairment. ${ }^{17}$ Preregistration and tertiary level education of community pharmacy personnel should include specific learning outcomes on safety and communication, and information about the range of assistive devices available to support older people with sensory impairment. Those learning outcomes should be translated into continuing professional education requirements. For example, a recent collaboration between different stakeholders in the UK developed guidance for pharmacy staff about the provision of pharmacy services for people with sight loss. ${ }^{15}$ In the USA, a Continuing Pharmacy Education package was developed to raise pharmacist awareness about patients with sight impairment. ${ }^{31}$ Further exploration is needed of role performance indicators.

\section{Strengths and limitations}

This study used a multiple methods approach. Data saturation was achieved with the interviews of service users and providers. The low response rate with the survey limited the generalisability of the results. The recruitment of patient participants through third parties might have resulted in the participation of more engaged patients. However, snowballing recruitment was also adopted and may have increased the diversity of patient participants. Community pharmacy personnel were recruited via the CPS which has almost universal coverage in Scotland.

\section{CONCLUSIONS}

This is the first study to explore and compare the pharmaceutical care needs of older people with sensory impairment from both a service user and service provider perspective. This study adds to the very limited evidence of the provision of pharmaceutical care for older patients with a sensory impairment.
This patient population experiences numerous challenges throughout their pharmaceutical care journey and, as such, are at considerable risk of iatrogenic harm. Community pharmacy personnel are aware of some of these challenges that their patients experience but need support to provide person-centred, safe and effective care to this population particularly in terms of communication and safety.

This study identified patient, pharmacy and organisational factors which influence (both positively and negatively) pharmaceutical care. The results will be used to develop and evaluate interventions through further research to achieve safe and effective pharmaceutical care for older people with sensory impairment.

Acknowledgements We thank all the community pharmacy personnel and people with sensory impairment who participated in these interviews. We also thank Community Pharmacy Scotland for assisting with the dissemination of information as part of the recruitment process. We thank the members of the project advisory group, which included representatives from Action on Hearing Loss, Sight Action and Age Scotland, who provided advice and guidance to the project team. Ms Yolanda Strachan, Age Scotland, was one of the original co-applicants for this project and has asked to be acknowledged in this section rather than as a co-author of this report to reflect her level of input.

Contributors All authors made significant contributions to the manuscript, contributed to the manuscript refinement and are responsible for its content. All authors read and approved the final manuscript. NA contributed towards data coding, analysis, interpretation and manuscript production. LM, AS and KMS contributed to the scientific development, conduct, interpretation of the study and revisions. AJT contributed towards data coding, analysis interpretation and revision. KK conducted all interviews, data collection, interpretation of the study and revisions. TK contributed to the scientific development of the study, reviewed the data analysis and its interpretation and the editing and development of the manuscript. MW led the scientific development and interpretation of the study, manuscript production and revisions.

Funding We are grateful to the Chief Scientist Office, Scottish Government which funded this study (CZH/4/1113).

Competing interests None declared.

Patient consent Not required.

Ethics approval University of Dundee Research Ethics Committee (January 2016: Ethics Review reference number 15187).

Provenance and peer review Not commissioned; externally peer reviewed.

Data sharing statement No additional data are available. All data related to this study are included in this submission, either in tables in the manuscript or in supplementary files.

Open access This is an open access article distributed in accordance with the Creative Commons Attribution Non Commercial (CC BY-NC 4.0) license, which permits others to distribute, remix, adapt, build upon this work non-commercially, and license their derivative works on different terms, provided the original work is properly cited, appropriate credit is given, any changes made indicated, and the use is non-commercial. See: http://creativecommons.org/licenses/by-nc/4.0/.

\section{REFERENCES}

1. Brennan M, Horowitz A, Su YP. Dual sensory loss and its impact on everyday competence. Gerontologist 2005;45:337-46.

2. Mathers CD, Loncar D. Projections of global mortality and burden of disease from 2002 to 2030. PLoS Med 2006;3:e442.

3. Dalton DS, Cruickshanks KJ, Klein BE, et al. The impact of hearing loss on quality of life in older adults. Gerontologist 2003;43:661-8.

4. Gopinath B, Hickson L, Schneider J, et al. Hearing-impaired adults are at increased risk of experiencing emotional distress and social engagement restrictions five years later. Age Ageing 2012;41:618-23.

5. La Grow S, Alpass F, Stephens C, et al. Factors affecting perceived quality of life of older persons with self-reported visual disability. Qual Life Res 2011;20:407-13. 
6. Crews JE, Campbell VA. Vision impairment and hearing loss among community-dwelling older Americans: implications for health and functioning. Am J Public Health 2004;94:823-9.

7. Scholes S, Faulding S, Mindell J. Health Survey for England 2013, chapter 5: use of prescribed medicines. http://digital.nhs.uk/ catalogue/PUB16076 (accessed Mar 2018).

8. Hughes CM, Cooper JA, Ryan C. Going beyond the numbers - a call to redefine polypharmacy. Br J Clin Pharmacol 2014;77:915-6.

9. Allemann SS, van Mil JW, Botermann L, et al. Pharmaceutical care: the PCNE definition 2013. Int J Clin Pharm 2014;36:544-55.

10. United Nations Convention on the Rights of Persons with Disabilities (UN CRPD). Article 25 - Health. https://www.un.org/development/ desa/disabilities/convention-on-the-rights-of-persons-withdisabilities/article-25-health.html (accessed Mar 2018).

11. McCann RM, Jackson AJ, Stevenson M, et al. Help needed in medication self-management for people with visual impairment: case-control study. Br J Gen Pract 2012;62:e530-e537.

12. Zhi-Han L, Hui-Yin Y, Makmor-Bakry M. Medication-handling challenges among visually impaired population. Arch Pharm Pract 2017;8:8-14.

13. Kentab BY, Al-Rowiali KZ, Al-Harbi RA, et al. Exploring medication use by blind patients in Saudi Arabia. Saudi Pharm J 2015;23:102-6.

14. Ferguson M, Liu M. Communication needs of patients with altered hearing ability: Informing pharmacists' patient care services through focus groups. J Am Pharm Assoc 2015;55:153-60.

15. Barnett N, El Bushra A, Huddy H, et al. How to support patients with sight loss in pharmacy. Pharm J 2017 http://www.pharmaceuticaljournal.com/pharmacy-learning-centre/how-to-support-patientswith-sight-loss-in-pharmacy/20203346.article (accessed Mar 2018).

16. Killick K, Macaden L, Smith A, et al. A scoping review of the pharmaceutical care needs of people with sensory loss. Int J Pharm Pract. In Press. 2018.

17. Ferguson MC, Shan L. Survey evaluation of pharmacy practice involving deaf patients. J Pharm Pract 2016;29:461-6.

18. Stewart $\mathrm{C}$, Kelly $\mathrm{M}$, Herrera $\mathrm{H}$. A survey study of community pharmacy practices in providing communication support to patients with sensory impairment. Int J Pharm Pract 2017;25(Suppl 1):49.
19. Community Pharmacy Scotland. http://www.communitypharmac yscotland.org.uk/ (accessed Mar 2018).

20. Shaghaghi A, Bhopal RS, Sheikh A. Approaches to recruiting 'hardto-reach' populations into re-search: a review of the literature. Health Promot Perspect 2011;1:86-94.

21. Campbell JL, Quincy C, Osserman J, et al. Coding in-depth semi-structured interviews: problems of unitization and intercoder reliability and agreement. Sociol Methods Res 2013;42:294-320.

22. Braun V, Clarke V. Using thematic analysis in psychology. Qual Res Psychol 2006;3:77-101.

23. Saldana J. The coding manual for qualitative researchers. 3rd ed. London: Sage Publications Ltd, 2016.

24. NHS National Services Scotland. Chronic medication service. https:// nhsnss.org/services/practitioner/pharmacy/pharmacy-services/ chronic-medication-service/ (accessed Mar 2018).

25. Grills A, MacDonald A. An assessment of the pharmaceutical needs of the blind and partially sighted in Dumfries and Galloway. Pharm $J$ 1997;259:381-4.

26. The Scottish government. Prescription for excellence: a vision and action plan for the right pharmaceutical services through integrated partnerships and innovation. 2013. http://www.gov.scot/Resource/ 0043/00434053.pdf (accessed Mar 2018).

27. Steinberg AG, Barnett S, Meador HE, et al. Health care system accessibility. Experiences and perceptions of deaf people. J Gen Intern Med 2006;21:260-6.

28. Davis A. Sensory impairment. In: Annual report of the chief medical officer, surveillance volume: on the state of public's health. 2012:56-7 (accessed Mar 2018).

29. Bernsten C, Björkman I, Caramona M, et al. Improving the wellbeing of elderly patients via community pharmacy-based provision of pharmaceutical care: a multicentre study in seven European countries. Drugs Aging 2001;18:63-77.

30. Bate P, Robert G. Experience-based design: from redesigning the system around the patient to co-designing services with the patient. Qual Saf Health Care 2006;15:307-10.

31. Orrico KB. Caring for visually impaired patients. J Am Pharm Assoc 2013;53:e142-e150. 